\title{
BMJ Open Cost-effectiveness of first-line erlotinib in patients with advanced non-small- cell lung cancer unsuitable for chemotherapy
}

\author{
Iftekhar Khan, ${ }^{1,2}$ Stephen Morris, ${ }^{2}$ Allan Hackshaw, ${ }^{1}$ Siow-Ming Lee ${ }^{3}$
}

To cite: Khan I, Morris S, Hackshaw A, et al. Costeffectiveness of first-line erlotinib in patients with advanced non-small-cell lung cancer unsuitable for chemotherapy. BMJ Open 2015;5:e006733.

doi:10.1136/bmjopen-2014006733

\section{- Prepublication history} and additional material is available. To view please visit the journal (http://dx.doi.org/ 10.1136/bmjopen-2014006733).

The clinical results from this were first presented as an oral presentation at ASCO 2011.

Received 24 September 2014 Revised 11 May 2015 Accepted 12 June 2015

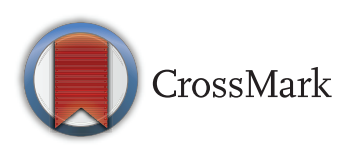

${ }^{1}$ CRUK \& UCL Cancer Trial Centre, University College London, London, UK ${ }^{2}$ Department of Applied Health Research, University College London, London, UK ${ }^{3}$ University College London Hospital/UCL Cancer Institute, London, UK

Correspondence to Dr Iftekhar Khan; Iftekhar.khan@ucl.ac.uk

\section{ABSTRACT}

Objective: To assess the cost-effectiveness of erlotinib versus supportive care (placebo) overall and within a predefined rash subgroup in elderly patients with advanced non-small-cell lung cancer who are unfit for chemotherapy and receive only active supportive care due to their poor performance status or presence of comorbidities.

Setting: Between 2005 and 2009, a total of 670 patients with non-small cell lung cancer (NSCLC) were randomised across 78 hospital sites (centres) in the UK.

Participants: 670 patients with pathologically confirmed stage IIIb-IV NSCLC, unfit for chemotherapy, predominantly poor performance status ( $>2$ on Eastern Cooperative Oncology Group, ECOG) and estimated life expectancy of at least 8 weeks. Patients were followed until disease progression or death, including a subgroup of patients who developed first cycle rash.

Interventions: Patients were randomised (1:1) to receive best supportive care plus oral placebo or erlotinib (150 mg/day) until disease progression, toxicity or death.

Primary outcome: Overall survival (OS).

Secondary outcomes: Progression-free survival (PFS), tumour response and quality adjusted life years (QALY), including within prespecified subgroups.

Results: The mean incremental cost per QALY in all patients was £202 571/QALY. The probability of costeffectiveness of erlotinib in all patients was $<10 \%$ at thresholds up to $£ 100000$. However, within the rash subgroup, the incremental cost/QALY was $£ 56770 /$ QALY with a probability of cost-effectiveness of about $80 \%$ for cost-effectiveness thresholds between $£ 50000$ to $£ 60000$.

Conclusions: Erlotinib has about $80 \%$ chance of being cost-effective at thresholds between $£ 50000-$ $£ 60000$ in a subset of elderly poor performance patients with NSCLC unfit for chemotherapy who develop first cycle (28 days) rash. Erlotinib is potentially cost-effective for this population, for which few treatment options apart from best supportive care are available.

Trial registration number: (ISCRTN): 77383050.

\section{Strengths and limitations of this study}

- A randomised controlled trial designed prospectively to assess the cost-effectiveness of erlotinib in a predefined subgroup. Rash is used as a retrospective surrogate marker to select patients to continue erlotinib treatment after 4 weeks trial of treatment.

- Quality of Life Data were obtained prospectively up to and beyond progression. In most trials Quality of Life is collected only up to progression.

- More than $99 \%$ of patients died, therefore, the uncertainty in modelling future health benefits is largely absent in this analysis.

- This is an important contribution in an area where there is limited data on the cost-effectiveness of cancer treatments in elderly patients.

- The potential economic impact of erlotinib use in the $60 \%$ of patients who develop rash is likely to be significant for the UK National Health Service (NHS).

\section{INTRODUCTION}

Lung cancer, the leading cause of cancerrelated death, accounts for nearly 1.4 million deaths worldwide annually, with a yearly incidence of over 41000 in the UK, ${ }^{12}$ of which $80 \%$ are non-small cell lung cancer (NSCLC). ${ }^{3}$ In the USA, the mean monthly cost of treating patients with lung cancer was estimated at £1669 (no active treatment, about $£ 1669$; $£ 1=\$ 1.61$ US) and $£ 5814$ (chemoradiotherapy). ${ }^{4}$ In the UK, the total annual cost of treating lung cancer in 2012 equated to about $£ 2.4$ billion $^{5}$ - the yearly average cost per patient was $£ 9071$. This compares with $£ 2756$ for bowel cancer, $£ 1584$ for prostate cancer and $£ 1076$ for breast cancer. The costs associated with lung cancer are therefore a significant economic burden on healthcare systems worldwide. 
It is estimated about $30 \%$ of patients with advanced NSCLC do not receive any cytotoxic treatment ${ }^{6}$ because of poor performance status and/or multiple medical comorbidities. The National Institute for Health and Care Excellence (NICE) does not recommend erlotinib treatment for epidermal growth factor receptor (EGFR) mutation-negative patients. ${ }^{7}$ NICE previously recommended erlotinib as an alternative treatment to docetaxel for patients with good performance status who have tried at least one chemotherapy treatment. ${ }^{8}$ The current cost-effectiveness thresholds (willingness to pay) proposed by NICE are $£ 20000-£ 30000$ per QALY; however, for special cases, such as end-of-life treatments, supplementary guidance was issued, which suggested a higher threshold to $£ 50000 /$ QALY.

The recently published TOPICAL trial ${ }^{9}$ was a randomised (1:1), double blind, phase III multicentre trial conducted in the UK comparing erlotinib (ER) with placebo in predominantly elderly patients receiving best supportive care considered unfit for chemotherapy because of poor performance status and/or multiple medical comorbidities, including renal impairment. The median overall survival (OS) was 3.7 versus 3.6 months for erlotinib versus placebo; $(\mathrm{HR}=0.94 ; 95 \%$ CI 0.81 to 1.1 ; $\mathrm{p}$ value $=0.46) .{ }^{9}$ However, in prespecified subgroup analyses in patients who developed rash after about 28 days (1 cycle), OS improved with erlotinib; (OS HR=0.76; 95\% CI 0.63 to $0.92 ; \mathrm{p}$ value $=0.0058$ and progression-free survival (PFS) $\mathrm{HR}=0.66$; $95 \%$ CI 0.54 to 0.80 ; $\mathrm{p}$ value $<0.001$ ); median OS of 2.9 versus 6.2 months.

About 178/302 (60\%) of patients in the TOPICAL trial developed rash with erlotinib in the first treatment cycle and $>70 \%$ developed rash at any time, consistent with what has been reported elsewhere. ${ }^{9}$ In the UK alone, about $30-40 \%$ of patients with NSCLC are unfit for chemotherapy ${ }^{9}$ therefore, assuming a worldwide advanced NSCLC incidence of approximately 1 million, ${ }^{2}$ the potential economic impact of erlotinib use in the $60 \%$ of treated patients who develop rash is likely to be significant. We present the results of a cost-effectiveness analysis of erlotinib versus placebo overall and within a predefined subgroup of patients who develop rash within the first (28 days) of treatment. The rationale for erlotinib treatment benefit in patients who develop rash has been discussed extensively. ${ }^{9-11}$

\section{PATIENTS AND METHODS}

\section{Patients}

The patient population included in this analysis were newly diagnosed stage IIIb-IV (pathologically confirmed) patients with NSCLC who were chemotherapy naïve with no symptomatic brain metastases, and deemed unsuitable for chemotherapy by treating physicians based on the Eastern Cooperative Oncology Group (ECOG) performance status ( $\mathrm{PS} \geq 2$ ) and/or multiple medical comorbidities including renal impairment.
Patients were followed up until progression or death. The primary end point was OS; secondary end points were PFS, safety and health-related quality of life (HRQoL). PFS was defined as the time between randomisation and progression or death (whichever occurred first). Progression was based using the response evaluation criteria in solid tumours (RECIST). Prespecified subgroup analyses included whether or not patients developed treatment-related rash within the first 28 days (first-cycle rash), gender and histology. HRQoL using EuroQoL EQ-5D-3L (EQ-5D) was assessed monthly. Randomised patients who took study medication were included in this analysis. Rash was assessed using a subjective 5 point scale graded from: no rash (grade 0), erythema alone (grade A), erythema with papules (grade B), erythema with papules and pustules (grade C) and erythema with papules and confluent pustules (grade D). Rash is a common side effect of erlotinib. In this analysis we included patients with all rash grades, regardless of expected relationship with study treatment. Patients considered to have an outcome of rash were those recorded to have a rash score of $\mathrm{A}-\mathrm{D}$. Those with a grade 0 were considered to have no rash.

\section{Treatments}

The main comparison is patients randomised to erlotinib who developed first cycle rash (ER) versus placebo. All patients were allowed to receive immediate or delayed palliative chest radiotherapy and/or radiotherapy to metastatic sites as appropriate.

Patients received erlotinib $(150 \mathrm{mg})$ or matching placebo daily until progression. Dose reductions to $100 \mathrm{mg}$ or $50 \mathrm{mg}$ were allowed. Patients who took erlotinib but did not develop rash (ENR) are discussed in the sensitivity analyses.

\section{Costs/resources}

The costs relevant for this analysis were costs from drug (erlotinib), radiotherapy, additional anticancer treatments (eg, other tyrosine-kinase inhibitor (TKI) or mono chemotherapy), patient management (hospital clinic visits, day cases and hospital admissions) and managing important treatment related-adverse events (AEs; eg, diarrhoea and rash). Resource use was collected monthly on the case report forms (CRF). Unit prices were taken from hospital pharmacy records, published NICE reports ${ }^{7} 8{ }^{12}$ (where available), published literature, ${ }^{13}{ }^{14-16}$ National Health Service (NHS) reference $\operatorname{costs}^{17}$ and the British National Formulary (BNF, 2012) ${ }^{18}$ without adjusting for inflation. Costs were estimated in UK (£) sterling. No discounting was applied to costs or health benefits $<1$ year; discounting at $3.5 \%$ per annum was applied in the second year; nearly all patients $(>99 \%)$ progressed/died by 2 years.

\section{Drug use}

Erlotinib price was set at $£ 54.37 /$ tablet for $150 \mathrm{mg}$, $£ 44.12$ for $100 \mathrm{mg}$ and $£ 25.21$ for $50 \mathrm{mg} .{ }^{78}$ Drug use was 
determined from the recorded number of tablets dispensed and returned. Drug cost per patient was estimating by multiplying the duration of erlotinib use by the unit price. For placebo, drug cost was set to zero. Where additional treatments were given (for palliation), unit costs were identified.

\section{Supportive care}

The mean price of palliative radiotherapy (including planning) for advanced stage patients with NSCLC was assumed to be $£ 120$ per visit using NHS reference prices (2011-2012). ${ }^{17}$ The total radiotherapy cost is computed by multiplying the duration of therapy by the unit price. Costs for additional anticancer treatments were included in the analysis; the daily price of gemcitabine/carboplatin was assumed to be $£ 47.50$; for vinorelbine it was $£ 19.18 .{ }^{14} 15$

\section{Clinic visits/admissions}

Resource use (hospital and additional clinic visits, day cases, night hospital stays) were recorded on the CRFs. Only hospitalisations from AEs that were recorded as definitely/probably/possibly treatment related were used to compute costs of hospital overnight stays. The price/day for a clinic visit was $£ 100$; day case was $£ 670$ and an additional $£ 730$ for overnight stay (ie, $£ 1400$ including admission). ${ }^{17}$

\section{Adverse events}

Clinically important grade 3 and above serious adverse events (SAEs) with $>5 \%$ frequency were: rash, diarrhoea and dyspnoea; ${ }^{9}$ other toxicity rates $(\leq 1 \%)$ were similar between treatments and expected cost differences were negligible. Patients with any grade (maximum AE grade) were included; it was assumed that palliative treatment was taken, even if AEs were milder. For rash and diarrhoea, the costs per day were set to $£ 4.30$ and $£ 8.59$, respectively; ${ }^{16}$ the cost of morphine at $15 \mathrm{~mL} /$ day is about £0.22; steroid use (dexamethasone) based on $£ 13.80$ per 100 tablets with standard doses of 3 tablets of 4-8 mg per day was set at $£ 0.42$ /daily dose; with salbutamol assuming $£ 0.13 /$ daily dose. ${ }^{18}$

Duration of AEs was computed from their start/end dates; daily unit prices of medications for treating AEs (following UK practice) were computed based on a monthly course. ${ }^{18}$ The total cost (per patient) was computed by adding component costs: drug cost, supportive care, palliative radiotherapy (RT), additional clinic visits, hospital day cases, hospital admissions and treatments for SAEs.

\section{Utilities}

The EQ-5D was used to construct health utilities for economic evaluation. The EQ-5D consists of 5 scales (mobility, self-care, usual activities, pain/discomfort and anxiety/depression) collected from baseline until progression/death. Responses were converted into utilities using a UK social tariff based on the time trade off method. ${ }^{19}$ Missing utility data were handled through multiple imputation (MI) techniques. Responses to EQ-5D were captured on paper CRFs during clinic assessments (monthly in year 1 and 6 monthly thereafter).

\section{Cost-effectiveness analysis}

A patient level (Partitioned Survival) cost utility analysis was undertaken. The OS, PFS and post-progression survival (PPS) were determined for each patient. Mean EQ-5D utilities over time were estimated for pre and postprogression periods and multiplied by corresponding survival times to derive QALYs for each patient. The total costs were then modelled to derive mean costs. Finally, mean incremental costs, QALYs and the incremental cost-effectiveness ratio (ICER) were derived.

\section{Sensitivity analysis}

One way sensitivity analyses were carried out, varying resource use by $\pm 20 \%$. In addition, a probabilistic sensitivity analysis (PSA) was carried out using Monte-Carlo simulation (10000 simulations) using multivariate methods. ${ }^{20}$

For each data set simulated, the incremental net benefit (INB) was computed using the relationship: $\mathrm{INB}=\lambda^{*} \Delta_{\mathrm{E}}-\Delta_{\mathrm{C}}$, where $\lambda$ is the CE threshold, $\Delta_{\mathrm{E}}$, the mean incremental effect and $\Delta_{\mathrm{C}}$ the mean incremental cost. The proportion of INBs above or below varying values of $\lambda$ (ranging from $£ 1000$ to $£ 100000$ ) was used to approximate the probability of cost-effectiveness.

\section{Statistical analysis}

A patient level statistical modelling approach was used to determine mean incremental costs and effects. Mean OS and PFS were determined using Kaplan-Meier methods. Utilities were modelled using linear mixed effects models for repeated measures adjusting for baseline and for whether the observed utility occurred preprogression or postprogression. Mean incremental QALYs were subsequently derived. No extrapolation of OS and PFS was carried out ( $>99 \%$ of patients had died at the time of analysis).

Total costs were modelled using a generalised linear model assuming a gamma distribution to derive the incremental mean costs. ${ }^{21}$ Since costs are positive $(>0)$ and skewed, a gamma distribution was considered to be adequate, ${ }^{21} 22$ although this requires a small increment of 0.001 to be added if costs are zero for modelling purposes. MI methods with a maximum of three data sets were used for missing data. The resulting SEs from MI were used to revise estimates of the ICER in sensitivity analyses. Simulation of cost and effects for PSA was carried out using multivariate methods ${ }^{23}$ by generating data from a Normal Copula. ${ }^{24}$ All analyses were conducted using SAS V.9.3. 


\section{RESULTS}

\section{Baseline characteristics}

Between 2005 and 2009, a total of 670 (350 erlotinib; 320 placebo) patients were randomised across 78 centres in the UK. From 334 versus 313 patients who took study treatment, a prespecified subgroup of 302 erlotinib versus 278 placebo was evaluable for first cycle rash (figure 1 CONSORT). The main comparison of interest in this CE analysis is the subgroup ER $(n=178)$ versus placebo $(n=278)$, since overall there were no differences between erlotinib and placebo; 178/302 (59\%) developed rash in the first cycle (ER group) and 124/302 (41\%) took erlotinib and did not develop rash in the first cycle (Erlotinib non-rash (ENR) group); 5/313 (2\%) on placebo had rash. Patients with ENR were included in a sensitivity analysis. Baseline characteristics were generally similar between groups (table 1). Although there appeared to be a difference for smoking status (except never-smokers), a multivariate analysis ${ }^{9}$ showed that overall survival was similar between ex-smokers and never-smokers (HR 0.98). Also, the efficacy of erlotinib may be reduced in patients who currently smoke, but the absolute difference of $24 \%$ versus $37 \%(\mathrm{p}=0.003)$ does not materially impact the estimate of the QALY and ICER; and, furthermore, we have shown that efficacy is improved in the erlotinib-rash group.

\section{Costs/resources}

Erlotinib was taken as $150 \mathrm{mg}$ tablets by about $83 \%$ of patients, without any dose reductions; $15 \%$ and $2 \%$ of patients reduced dose to $100 \mathrm{mg}$ and $50 \mathrm{mg}$, respectively. In the rash subgroup this was $79 \%$ for $150 \mathrm{mg}$ tablets, $20 \%$ for $100 \mathrm{mg}$ and $1 \%$ for $50 \mathrm{mg}$ tablets, respectively. Hence, after taking into account dose reductions and dose delays, the mean cost of erlotinib (table 2) was $£ 6863$ overall and £7544 in the rash subgroup. For ER versus placebo, additional chemotherapy/TKI costs after progression on the erlotinib arm $(n=7)$ and placebo group $(\mathrm{n}=5)$ were $£ 182$ versus $£ 270$, respectively (mean difference $£ 88$, $p$ value $=0.852$ ); mean costs for palliative radiotherapy were $£ 302$ versus $£ 235$ ( $p$ value $=0.0449$ );

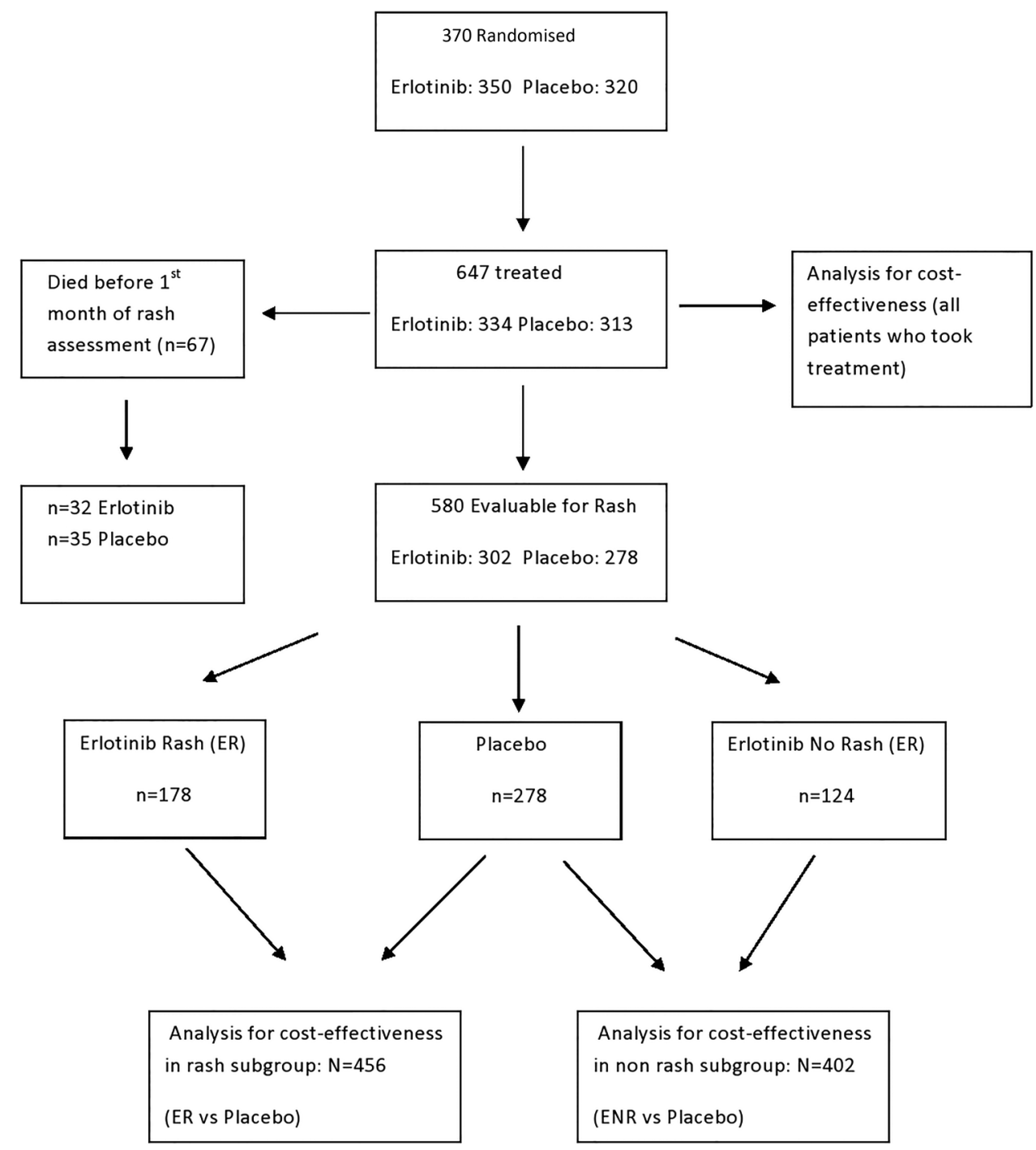

Figure 1 Consort diagram. 
Table 1 Summary of baseline characteristics ${ }^{*}$

\begin{tabular}{|c|c|c|c|c|c|c|}
\hline & \multicolumn{2}{|l|}{ Overall } & \multirow[b]{2}{*}{ p Value } & \multicolumn{2}{|l|}{ Rash subgroup } & \multirow[b]{2}{*}{ p Value } \\
\hline & Erlotinib (N=350) & Placebo $(\mathrm{N}=320)$ & & Erlotinib (N=178) & Placebo (N=278) & \\
\hline \multicolumn{7}{|l|}{ Age } \\
\hline Range & $(45-91)$ & $(51-91)$ & & $(51-91)$ & $(45-91)$ & \\
\hline \multicolumn{7}{|l|}{ Gender } \\
\hline Female & 135 (39\%) & $126(39 \%)$ & 0.833 & 69 (39\%) & $108(39 \%)$ & 0.984 \\
\hline $0-1$ & $54(15 \%)$ & $53(16 \%)$ & 0.692 & $37(21 \%)$ & $50(18 \%)$ & 0.459 \\
\hline 2 & 194 (55\%) & $185(56 \%)$ & 0.539 & 103 (58\%) & $165(59 \%)$ & 0.756 \\
\hline 3 & 102 (25\%) & $90(27 \%)$ & 0.771 & $38(21 \%)$ & $63(23 \%)$ & 0.741 \\
\hline \multicolumn{7}{|l|}{ Cell type } \\
\hline Adenocarcinoma & $133(38 \%)$ & $123(38 \%)$ & 0.908 & $63(35 \%)$ & 103 (37\%) & 0.718 \\
\hline Smoker & $124(35 \%)$ & $119(37 \%)$ & 0.631 & $43(24 \%)$ & $104(37 \%)$ & 0.003 \\
\hline Ex-smoker & 207 (59\%) & $183(57 \%)$ & 0.608 & $122(69 \%)$ & 158 (57\%) & 0.012 \\
\hline Never smoked & $19(5 \%)$ & $18(6 \%)$ & 0.911 & $13(7 \%)$ & $16(6 \%)$ & 0.509 \\
\hline
\end{tabular}

mean duration of radiotherapy was longer with erlotinib (2.5 vs 2.0 days; $p$ value $=0.3412$ ), although the proportion of patients who received radiotherapy was similar (table 2 and online supplementary table S1). More patients were hospitalised for treatment-related SAEs on ENR versus placebo: 22/178 (12\%) versus 15/278 (5\%); mean costs of treatment related SAEs were, therefore, $£ 356$ versus $£ 184$ and mean total costs were £9949 versus $£ 2058$, respectively.

\section{Efficacy}

The mean and SE for OS was $7.08(0.48)$ versus 6.41 (0.44) months. For PFS, this was 4.95 (0.36) versus 3.80 (0.29) months, respectively (erlotinib vs placebo). In the rash subgroup, OS was 9.08 (0.65) versus $6.91(0.43)$ months (table 2); and PFS was 6.22 (0.51) versus 4.19 (0.32) months for ER and placebo, respectively.

\section{Utilities and QALYs}

About $98 \%$ of EQ-5D forms were completed at baseline; patients alive at 1 year, $32 / 40(80 \%)$ versus $34 / 43(79 \%)$ had complete EQ-5D data for ER versus placebo, respectively. Missing data between groups were similar at other time points. As expected, HRQoL was better before progression (table 1): pre-progression utility was 0.6482 (0.009) versus $0.6438(0.011)$; for postprogression, mean (SE) utilities were $0.5517(0.016)$ versus $0.5760(0.0140)$ for erlotinib versus placebo, respectively. In the rash subgroup, EQ-5D utility improvement was higher prior to disease progression: $0.6407(0.017)$ versus $0.6193(0.015)$; mean difference was 0.0214 (95\% CI, -0.0122, 0.0651; $\mathrm{p}$ value $=0.3408$ ); for post-progression, mean (SE) was
$0.5548(0.0255)$ versus $0.5756(0.0200)$; mean difference of -0.0243 (95\% CI, $-0.0084,0.0429$; $p$ value $=0.5229)$ for ER versus placebo, respectively.

The mean QALY was 0.365 versus 0.3303 overall, yielding an incremental QALY of 0.035. In the rash subgroup, the mean QALY was, respectively, 0.467 versus 0.337 for ER versus placebo, yielding a statistically significant mean incremental QALY of 0.139 (95\% CI 0.0341 to $0.2359 ; \mathrm{p}$ value $=0.0070$ ), in favour of erlotinib. The improved QALY within the rash subgroup appears to be due to improved survival (table 3), notably PFS. Hence, the mean incremental cost was $£ 7090$ and an overall ICER of £202 571/QALY.

In the rash subgroup, the mean incremental cost was $£ 7891$ (95\% CI £6999-£8783), but with better HRQoL/ utility resulting with a base case ICER of $£ 56770 /$ QALY (table 4). The incremental cost excluding erlotinib cost was $£ 347$ and the ICER was $£ 2496 /$ QALY in the rash subgroup. The mean incremental net benefit (INB) for erlotinib is not realised until one is prepared to pay in excess of about $£ 202571$ overall and $£ 56770$ in the rash subgroup.

\section{Assessing uncertainty}

Sensitivity analyses were conducted for the rash subgroup only. However, as part of the sensitivity analyses, the impact on the ICER was observed from patients in the ENR group who took erlotinib-since these patients would contribute towards the costs of erlotinib in practice,for at least one cycle.

Results from one way sensitivity analyses are shown in table 4 . The ICER was most sensitive to changes $( \pm 20 \%)$ in 
Table 2 Model inputs: unit prices for resource use and summary of costs

\begin{tabular}{|c|c|c|c|c|c|c|c|}
\hline \multirow[b]{2}{*}{ Costs Item } & \multirow[b]{2}{*}{$\begin{array}{l}\text { Estimated } \\
\text { unit price }(£)\end{array}$} & \multicolumn{2}{|l|}{ Rash subgroup } & \multirow[b]{2}{*}{$\begin{array}{l}\text { Difference } \\
\text { (p value) }(£)\end{array}$} & \multirow{2}{*}{$\begin{array}{l}\text { Overall }(£) \\
\text { Erlotinib } \\
\mathrm{N}=334 \\
\text { Mean (SE) }\end{array}$} & \multirow[b]{2}{*}{$\begin{array}{l}\text { Placebo/SC } \\
\mathrm{N}=313 \\
\text { Mean (SE) }\end{array}$} & \multirow[b]{2}{*}{ Difference } \\
\hline & & $\begin{array}{l}\text { Erlotinib (rash) }(£) \\
\mathrm{N}=178 \\
\text { Mean (SE) }\end{array}$ & $\begin{array}{l}\text { Placebo/SC (£) } \\
\mathrm{N}=278 \\
\text { Mean (SE) }\end{array}$ & & & & \\
\hline Erlotinib* & 54.37/tablet & $7544(764)$ & 0 & 7544 & $6863(674)$ & 0 & 8074 \\
\hline \multicolumn{8}{|l|}{ Supportive care: } \\
\hline Palliative RT† & 120/visit & $302(52)$ & $235(27)$ & $67(p=0.0449)$ & $350(48)$ & 242 (39) & 108 \\
\hline Additional treatment $\ddagger$ & See note c & $182(65)$ & 270 (99) & $-88(p=0.85)$ & $190(59)$ & $264(76)$ & -74 \\
\hline \multicolumn{8}{|l|}{ Patient management: } \\
\hline Hospital clinic visit§ & 100/visit & $629(57)$ & $624(53)$ & $5(p=0.46)$ & $663(49)$ & $654(40)$ & 9 \\
\hline Hospital day case $\S$ & $670 /$ day case & $274(65)$ & $323(131)$ & $-49(p=0.69)$ & $285(71)$ & $356(95)$ & -71 \\
\hline Hospital admission§ & 730/night & 744 (163) & $475(134)$ & $269(p=0.0352)$ & 775 (149) & $534(122)$ & 241 \\
\hline Adverse events & See note e & $221(34)$ & $114(27)$ & $107(p<0.001)$ & $264(40)$ & $181(32)$ & 83 \\
\hline $\begin{array}{l}\text { Total mean cost }(\mathrm{SE})^{\star *} \\
(95 \% \mathrm{Cl})\end{array}$ & & $\begin{array}{l}9949(724) \\
(8530 \text { to } 11368)\end{array}$ & $\begin{array}{l}2058(185) \\
(1695 \text { to } 2420)\end{array}$ & $7891 p<0.001)$ & $9210(711)$ & 2121 (199) & 7089 \\
\hline Incremental Cost (SE) ${ }^{\star \star}$ & \multicolumn{4}{|c|}{$7891(614)(95 \% \mathrm{Cl} 6999$ to 8783$)$} & \multicolumn{3}{|c|}{7089 (589) (95\% Cl £5935 to £8243) } \\
\hline
\end{tabular}

${ }^{*}$ Cost as $£ 1631.53$ for 30 tablets ( $150 \mathrm{mg}$ tablet) or depending on dose; + Unit price based on national NHS tariff (NICE report 2011). ${ }^{7}$

†Palliative RT: Diagnosis and treatment of lung cancer update (2011). ${ }^{9}$

fOn the Placebo arm, 7 patients took additional chemotherapy (carboplatin/gemcitabine $(n=5)$ erlotinib $(n=2))$ after progression; on the erlotinib arm, patients took carboplatin $(n=2)$, vinorelbine $(n=2)$, Fragmin $(n=1){ }^{11}$

§Additional clinic visits and day visits irrespective of reason; unit prices taken from NHS reference costs 2010-2011; Hospital nights stayed as a result of treatment-related serious adverse events. ${ }^{13}$

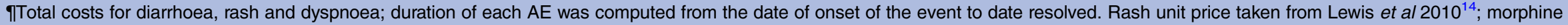

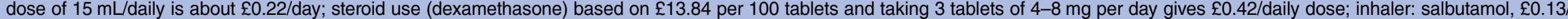
daily dose. ${ }^{15}$ Mean diarrhoea costs were $£ 14$ versus £2; mean rash costs were $£ 68$ versus $£ 14.90$ and mean dyspnoea costs were $£ 139$ versus $£ 97$.

${ }^{*}$ Determined using a generalised linear mixed model assuming gamma distributed costs for Erlotinib+Rash versus Placebo.NHS, National Health Service; NICE, National Institute for Health

and Care Excellence; RT, radiotherapy. 
Table 3 Model inputs: effectiveness measures

\begin{tabular}{|c|c|c|c|c|}
\hline & \multicolumn{2}{|l|}{ Overall } & \multicolumn{2}{|l|}{ Rash subgroup } \\
\hline & Erlotinib (N=334) & Placebo (N=313) & Erlotinib (N=178) & Placebo $(\mathrm{N}=278)$ \\
\hline Mean OS (months) & $7.08(0.48)$ & $6.41(0.44)$ & $9.08(0.65)$ & $6.91(0.43)$ \\
\hline Mean PFS (months) & $4.95(0.36)$ & $3.80(0.29)$ & $6.22(0.51)$ & $4.19(0.32)$ \\
\hline Mean PPS (months) & $2.13(0.250)$ & $2.61(0.236)$ & $2.86(0.41)$ & $2.72(0.27)$ \\
\hline $\mathrm{HR}(\mathrm{OS})$ & \multicolumn{2}{|l|}{0.92} & \multicolumn{2}{|l|}{0.76} \\
\hline (95\% Cl; p value) & \multicolumn{2}{|c|}{ (0.79 to $1.08 ; p$ value $=0.32)$} & \multicolumn{2}{|c|}{$(0.63$ to $0.92 ; p$ value $=0.005)$} \\
\hline HR (PFS) & \multicolumn{2}{|l|}{0.81} & \multicolumn{2}{|l|}{0.66} \\
\hline (95\% Cl; p value) & \multicolumn{2}{|c|}{$(0.70$ to $0.95 ; p$ value $=0.0102)$} & \multirow{2}{*}{\multicolumn{2}{|c|}{$(0.54$ to $0.80 ; p$ value $<0.0001)$}} \\
\hline Utilities & & & & \\
\hline Preprogression EQ-5D (mean, SE) & $0.6482(0.009)$ & $0.6438(0.011)$ & $0.6407(0.017)$ & $0.6193(0.015)$ \\
\hline Postprogression EQ-5D (mean, SE) & $0.5517(0.016)$ & $0.5760(0.014)$ & $0.5548(0.0255)$ & $0.5756(0.020)$ \\
\hline QALY (years)* & $0.365(0.0272)$ & $0.3303(0.0245)$ & $0.487(0.0432)$ & $0.3472(0.0260) \dagger$ \\
\hline Incremental QALY (mean SE)‡ & & $0.035(0.0163)$ & & $0.139(0.0113)$ \\
\hline
\end{tabular}

erlotinib costs, and utilities ranged from $£ 45821$ /QALY to $£ 67530$ /QALY. Most ICERs remained within 5\% of the base case after $\pm 20 \%$ adjustments. The mean ICER from PSA was $£ 57120$ with $5 \%$ and $95 \%$ quantiles ranging from $£ 29438$ to $£ 89550$. The estimated probability of costeffectiveness of erlotinib at CE thresholds between $£ 50000$ to $£ 60000$ was $80 \%$ (figure $2 \mathrm{~A}$ ). The CE plane (figure 2B) shows costs and benefits scattered in the north east quadrant where incremental effects are generally positive (erlotinib better), but with higher costs.

Patients who started erlotinib but did not develop rash after the first cycle (ENR) are considered to contribute towards drug costs during the first cycle. Patients with ENR who took erlotinib for at least one cycle had worse outcomes: OS and PFS HRs of 1.30 and 1.09 compared to placebo, with mean (SE) OS and PFS of $5.7(0.69)$ and $4.2(0.55)$ months, respectively. ${ }^{9}$ These patients are unlikely to continue erlotinib if rash is not observed within the first cycle (but may continue to receive SC). The mean cost of 28 days of erlotinib after accounting

Table 4 One way sensitivity analysis (rash subgroup)

\begin{tabular}{llr}
\hline Parameter & Variation (\%) & ICER (£) \\
\hline Base case & & 56768 \\
Erlotinib cost & -20 & 45821 \\
Radiotherapy costs & +20 & 67530 \\
& -20 & 56823 \\
Hospital admission costs & +20 & 55953 \\
Preprogression utility & +20 & 54018 \\
& -20 & 58758 \\
Postprogression utility & +20 & 97671 \\
& -20 & 48945 \\
Missing data adjustments* & +20 & 160019 \\
\hline *Using multiple imputation. & - & 49845 \\
& &
\end{tabular}

for dose delays and reductions was £1279. The total mean erlotinib cost was, therefore, $£ 8187$. Hence, after adjusting for all other costs (AEs, supportive care including RT, hospital visits) during the PFS period, the incremental cost and ICER increased to £9578 and £68 906, respectively, assuming an incremental QALY of 0.139.

The base case ICER was robust to differences in the models and methods of handling missing utility data. When MI was used, the ICER did not increase (table 4) beyond $£ 58400$ (range $£ 55452$ to $£ 58400$ ). The SEs of incremental costs and QALYs increased slightly from $£ 614$ to $£ 689$ and 0.0113 to 0.0145 , respectively, after taking into account missing data, using MI. A summary of ICER results are shown in table 5 .

\section{DISCUSSION}

In this trial in a population of patients with advanced NSCLC considered unfit for first-line chemotherapy, erlotinib did not show cost-effectiveness overall, however, it has potential for being cost-effective in patients who develop first cycle rash. At the time of our original report, ${ }^{9}$ previous phase III NSCLC clinical trials suggested a relationship between rash development and improved survival with TKI treatment. ${ }^{10} 2526$ This has since been confirmed in a meta-analysis of 33 trials (6798 patients) of NSCLC, from which the authors concluded that skin rash after EGFR-TKI treatment is an effective surrogate marker for predicting clinical outcomes. ${ }^{11}$ Our findings are also consistent with several other studies to report the relationship between TKIs/ EGFR antibodies and rash in different cancer types: Bonner, 2010 (head and neck cancer), ${ }^{27}$ Cunningham (2004) and Bokemeyer (2009) ${ }^{28}$ in colorectal cancer. ${ }^{29}$

The monthly cost of erlotinib treatment was $£ 1650^{9}$ and at least $£ 9112^{15}$ for six cycles. This trial demonstrated effects in our patients for whom few treatment 
Table 5 Summary of results from Cost Utility Analysis (CUA)

\begin{tabular}{|c|c|c|c|}
\hline Scenario & Incremental costs & Incremental effects & ICER (5th, 95th centile) \\
\hline Overall (base case)† & $£ 7090$ & 0.139 & $£ 202571$ \\
\hline \multicolumn{4}{|l|}{ Rash subgroup } \\
\hline Base case $\neq$ & $£ 7891$ & 0.139 & $£ 56770(£ 29438-£ 89550)$ \\
\hline Excluding erlotinib costs & $£ 347$ & 0.139 & $£ 2496$ (£1120-£3895) \\
\hline Including ENR 1st cycle drug costs§ & $£ 9578$ & 0.139 & $£ 68906$ (£44 165-£93 276 ) \\
\hline $\begin{array}{l}\text { †Erlotinib versus placebo }(n=647) \text {. } \\
\text { †ER versus placebo }(n=456) \text {. } \\
\text { §ER (including ENR first cycle erlotinib costs } \\
\text { ENR, Erlotinib non-rash; ICER, incremental c }\end{array}$ & $\begin{array}{l}\text { us placebo. } \\
\text { fectiveness ratio. }\end{array}$ & & \\
\hline
\end{tabular}

options are available apart from palliative radiotherapy. OS improved by a median of $>3$ months ${ }^{9}$; Improvements in HRQoL were also demonstrated with the EORTC-QLQC-30. ${ }^{9}$ Consequently, ER patients show a high probability (about $80 \%$ ) of being cost-effective at thresholds between $£ 50000$ and $£ 60000$; this threshold is advocated by supplementary NICE guidelines (in the UK) for end of life treatments. ${ }^{30}$ Moreover, at lower CE thresholds (eg, $£ 30000-£ 40000$ ), between $15 \%$ to $40 \%$ ( figure 2A) of our patients with NSCLC unfit for chemotherapy would have a cost-effective clinical benefit (ie, $>3$ months improvement in OS and improved HRQoL). With few treatment options available for these patients, this is an important finding.

From published trials, the cost/QALY of erlotinib ranges from $£ 18170$ to $£ 89377$ (incremental QALYs
Figure 2 Cost-effectiveness results. (A) Cost-Effectiveness Acceptability Curve (CEAC): ER versus Placebo/SC for rash subgroup. Note: Vertical reference lines are $C E$ threshold values of $£ 50000$ and the observed cost/QALY (£56 770). The horizontal reference line is 0.8. (B) Cost-Effectiveness Plane: $E R$ versus $P l a c e b o / S C$ (rash subgroup). Note: The first vertical reference line is 0 . The horizontal and second vertical reference lines are observed incremental effect (0.139) and observed incremental cost (£7891), respectively.
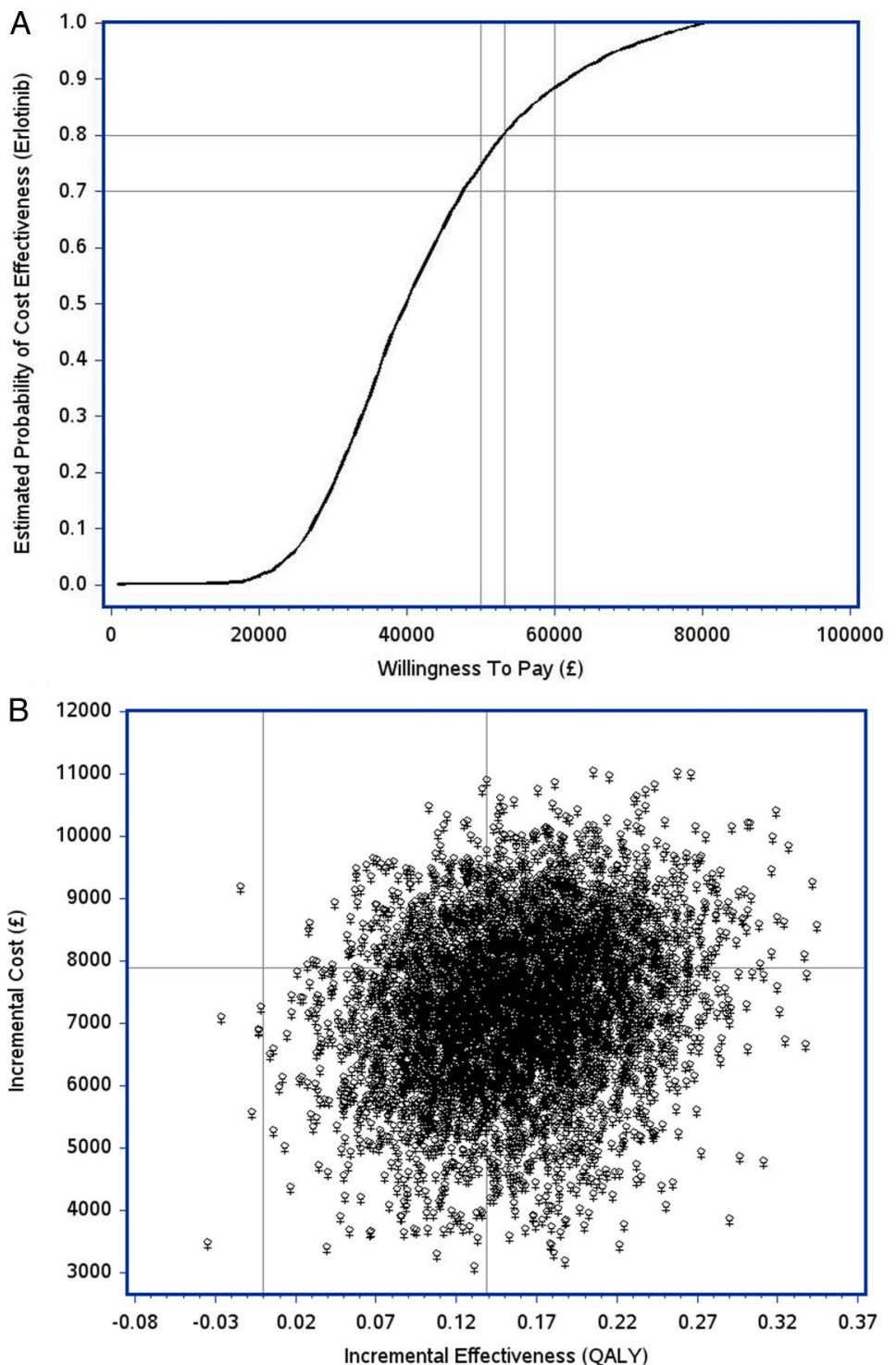
ranging from 0.175 to 1.4$).{ }^{16}{ }^{31-38}$ The observed QALY of 0.139 in ER patients was within this range. Reported ICERs in EGFR mutation +ve patients for other TKI therapies include: afatinib (ICERs between $\$ 45000$ ( $£ 23000$ ) to $\$ 75000$ ( $£ 38600$ )/QALY when compared with gefitinib, erlotinib and cisplatin/gemcitabine) ${ }^{39}$ for gefitinib £21 000 (vs gemcitiabine) to $£ 154000 /$ QALY (vs cisplatin/gemicitabine). ${ }^{40}$ However, these were in trials where patients were fit for standard chemotherapy. Non-TKI's such as pemetrexed reported ICERs between $£ 18672$ (vs BSC) ${ }^{41}$ to $£ 49000 /$ QALY (vs BSC). ${ }^{42}$ Crizotinib: ICERs ranging from about $£ 41500 /$ QALY $^{43}$ versus docetaxel to $\$ 216000 / \mathrm{QALY}$ when compared with pemetrexed. ${ }^{44}$

In a recent review of licenced treatments for NSCLC, it was highlighted that the elderly frail population were unrepresented in the majority of lung cancer trials and there remains considerable uncertainty in assessing the cost-effectiveness of treatments for this group of patients. ${ }^{45}$ Moreover, many of these trials did not report the cost/QALY, which would be helpful to decisionmakers. Interestingly, in a phase II erlotinib trial that did include elderly patients (fit for chemotherapy), the reported ICER was $€ 395400$ (£341 198/QALY). ${ }^{46}$

In the UK, the NICE supplementary guidance recognises that during end of life, the standard CE thresholds of $£ 20000-£ 30000$ per QALY gain may be inadequate. ${ }^{31} 47$ Two of these three criteria (short life expectancy and $\geq 3$ months improvement in survival) were satisfied. The third criteria: 'treatment is licensed or otherwise indicated for small patient populations' is not clearly satisfied; poor performance patients with NSCLC is not considered to be a 'small' patient population, despite this population being understudied. ${ }^{45}$ A 'small population' should not normally 'exceed 7000 new patients per year' or should be a 'small group within larger populations'. ${ }^{31}$

There are several strengths in our analyses. First, the TOPICAL trial was designed to prospectively record health economic data (eg, collecting resource use and utility data). Second, $>99 \%$ of patients died, therefore, the uncertainty in modelling future health benefits is largely absent. Third, utility data were collected until progression/death for all patients, thereby providing reliable mean postprogression utility estimates. Moreover, the EQ-5D utility data had good completion rates. Despite short survival times, costs and benefits were adequately captured.

A strength of this research is the low rate of EGFR mutation-positive tumours in our population. Currently, NICE recommended EGFR mutation-positive locally advanced or metastatic NSCLCs are treated with TKIs (gefitinib, erlotinib or afatinib) regardless of their performance status. It is possible that if ER patients were to be compared with a similar TKI, the ICER may be higher due to smaller differences between groups. Several other $\mathrm{CE}$ analyses exist that compared TKIs against placebo and were approved by NICE. ${ }^{31}$ It is also acknowledged that the analysis associated with rash was not based on a randomised comparison, so there may be some unknown confounders. We excluded deaths prior to the first cycle, but there were only $32(9 \%)$ versus $35(11 \%)$, which was not statistically significant, so unlikely to impact the results (because the survival time was $<1$ month in both treatment groups, the mean OS and PFS would be very similar). In addition, despite being statistically significant differences of $37 \%$ versus $24 \%$ in smoking status ( $p=0.003$ ) with median OS improvement of 2 months (erlotinib vs placebo) for ex-smokers and 1.4 months for current smokers, this did not translate to a meaningful QALY difference. The mean QALY was 0.11 and 0.15 ( $p=0.1580$ ) for current and ex-smokers, respectively. This suggests that the observed differences in smoking status did not appear to result in meaningful differences in the ICER: about $£ 47000$ (ex-smoker) to $£ 64000$ (current smoker) assuming the same incremental costs. These values of the ICER are within the range of the sensitivity analyses for the overall ICER.

The low EGFR mutation rate $(5 \%)$ was insufficient to establish the cost-effectiveness of erlotinib for poor performance patients whose tumours are EGFR mutation + ve. In any case, the target population in this trial may not require the (expense of) testing for EGFR mutation status and patients could stop taking erlotinib after the first cycle of rash. In this trial 124/302 (40\%) patients without rash continued to receive erlotinib. With the proposed treatment strategy, $40 \%$ of patients who took erlotinib would fall into this category and reduce the costs of erlotinib. Therefore, the cost saving could be much higher. Interestingly, all patients who tested positive for EGFR mutation developed rash.

Using the findings from our trial, we estimate that of 100 patients needing to be treated with erlotinib, 60 develop treatment-related rash, who are likely to benefit. The inclusion of the ENR costs for the first cycle (28 days) is justified because it represents a health resource consumed, although these patients are unlikely to improve. The ICER was $£ 56770$ /QALY excluding the cost of treating the $40 \%$ of non-rash patients for one cycle, and £68906/QALY including this cost. Both are within the range ( $£ 30000$ to $£ 154000 /$ QALY) of other estimates for gefitinib and afatinib as first-line therapy for EGFR-mutation-positive patients with NSCLC. Although the mean cost from 28 days of erlotinib use in patients with ENR has been included (when calculating the ICER of $£ 68906$ ), the ICER does not reflect the negative effects and poorer QoL (dis-utility) from erlotinib use in the patients with ENR group after first cycle of treatment. Therefore, the higher ICER of $£ 68906$, while reflecting total erlotinib usage, may not reflect the efficiency of erlotinib for the target population.

In conclusion, erlotinib offers a potentially costeffective treatment option for the subgroup of predominantly poor performance patients with NSCLC with EGFR wild-type tumours who develop first-cycle rash and who are considered unfit by clinicians to be treated with first-line chemotherapy. 
Correction notice The license of this article has changed since publication to CC BY 4.0.

Acknowledgements The authors are most grateful to all the participating patients, clinicians and local research staff for their helpful advice and comments throughout the trial. The authors are also grateful to Professor Mark Sculpher (University of York), the University College London Health Economics Group and all reviewers for their helpful comments to improve the final manuscript.

Contributors IK, S-ML and AH contributed to conception and design. S-ML and the participating site investigators were involved in provision of study materials or patients. IK contributed to statistical and cost-effectiveness analysis. IK, $\mathrm{AH}$ S-ML and SM contributed to data interpretation. IK contributed to manuscript writing. IK, AH, SM and S-ML approved the final version of manuscript.

Funding This was a Cancer Research UK Funded trial, Grant number C1438/ A4147; Free erlotinib was provided by Roche Pharmaceuticals. SML is part-funded by University College London Hospital Biomedical Research Centre.

Competing interests None declared.

Provenance and peer review Not commissioned; externally peer reviewed.

Open Access This is an Open Access article distributed in accordance with the terms of the Creative Commons Attribution (CC BY 4.0) license, which permits others to distribute, remix, adapt and build upon this work, for commercial use, provided the original work is properly cited. See: http:// creativecommons.org/licenses/by/4.0/

\section{REFERENCES}

1. Ferlay J, Shin HR, Bray F, et al. Estimates of worldwide burden of cancer in 2008: GLOBOCAN 2008. Int J Cancer 2010;127:2893-917.

2. UK CR. Lung cancer and smoking statistics (UK). London 2012 [12 July 2012]. http:/info.cancerresearchuk.orgcancerstats/

3. Toms JR. Cancer Stats Monograph 2004 Cancer Research UK. Cancer Incidence, survival and mortality in the UK and EU. London: Cancer Research UK, 2004.

4. Cipriano LE, Romanus D, Earle CC, et al. Lung cancer treatment costs, including patient responsibility, by disease stage and treatment modality, 1992 to 2003. Value Health 2011;14:41-52.

5. http://www.cancerresearchuk.org/cancer-nfo/news/archive/ pressrelease/2012-11-07-lung-cancer-price-tag (last accessed Jun 2013).

6. Davidoff AJ, Tang M, Seal B, et al. Chemotherapy and survival benefit in elderly patients with advanced non-small-cell lung cancer. J Clin Oncol 2010;28:2191-7.

7. https://www.nice.org.uk/guidance/gid-tag347/documents/erlotiniband-gefitinib-for-treating-nonsmallcell-lung-cancer-that-hasprogressed-following-prior-chemotherapy-review-of-ta162-and-ta175appraisal-consultation-document

8. Erlotinib for the treatment of non-small-cell lung cancer Issued: November 2008 last modified: December 2012 NICE technology appraisal guidance 162 guidance.nice.org.uk/ta162. http://guidance. nice.org.uk/TA162/Guidance/pdf/English

9. Lee SM, Khan I, Upadhyay S, et al. First-line erlotinib in patients with advanced non-small-cell lung cancer unsuitable for chemotherapy (TOPICAL): a double-blind, placebo-controlled, phase 3 trial. Lancet Oncol 2012;13:1161-70

10. Mohamed MK, Ramalingam S, Lin Y, et al. Skin rash and good performance status predict improved survival with gefitinib in patients with advanced non-small cell lung cancer. Ann Oncol 2005;16:780-5.

11. Liu HB, Wu Y, Lv TF, et al. Skin rash could predict the response to EGFR tyrosine kinase inhibitor and the prognosis for patients with non-small cell lung cancer: a systematic review and meta-analysis. PLOS ONE 2013;8:e55128.

12. Lung cancer: The diagnosis and treatment of lung cancer. National costing report: Lung cancer (April 2011). http://www.nice.org.uk/ guidance/cg121/evidence/cg121-lung-cancer-full-guidelineappendix-102

13. Diagnosis and treatment of lung cancer update (2011). Palliative RT page 135: Table A4.11. http://www.nice.org.uk/guidance/cg121/ evidence/cg121-lung-cancer-full-guideline-appendix-102

14. Brown T, Boland A, Bagust A, et al. Gefitinib for the first-line treatment of locally advanced or metastatic non-small cell lung cancer (NSCLC): a single technology appraisal. LRiG, The University of Liverpool, 2009.
15. McLeod C, Bagust A, Boland A, et al. Erlotinib for the treatment of relapsed non-small cell lung cancer. Health Technol Assess 2009; 13(Suppl 1):41-7.

16. Lewis G, Peake M, Aultman R, et al. Cost-effectiveness of erlotinib versus docetaxel for second-line treatment of advanced non -smallcell lung cancer in the United Kingdom. J Int Med Res 2010;38:9-21.

17. NHS reference costs 2010-2011. https://www.gov.uk/government/ publications/2010-11-reference-costs-publication (17 Nov 2011).

18. Joint Formulary Committee. British National Formulary. 1st edn. London: BMJ Group and Pharmaceutical Press, 2012.

19. Dolan P. Modeling valuations for EuroQol health states. Med Care 1997;35:1095-108.

20. Briggs A, Sculpher M, Claxton K. Decision modelling for health economic evaluation (handbooks for health economic evaluation). Oxford University Press, 2006.

21. Mihaylova B, Briggs A, O'Hagan A, et al. Review of statistical methods for analysing healthcare resources and costs. Health Econ 2011;20:897-916.

22. Khan I. Design and analysis of clinical trials for economic evaluation \& re-imbursement. Chapman \& Hall Publications. In press. https:// www.crcpress.com/product/isbn/9781466505476

23. Nelson RB. An introduction to Copulas. Springer Texts, 2006

24. Khan I. Probabilistic sensitivity analyses for clinical trials of costeffectiveness using the method of copulas: a comparison of simulation methods. Oral presentation at the Society for Clinical Trials, 2014. http://www.sctweb.org/public/search/detail.cfm? ID=A7C6951B-EAD9-AA85-9BD63CC8386DE3E1

25. Gatzemeier U, von Pawel J, Vynnychenko I, et al. First-cycle rash and survival in patients with advanced non-small-cell lung cancer receiving cetuximab in combination with first-line chemotherapy: a subgroup analysis of data from the FLEX phase 3 study. Lancet Oncol 2011:12:30-7.

26. Wacker B, Nagrani T, Weinberg J, et al. Correlation between development of rash and efficacy in patients treated with the epidermal growth factor receptor tyrosine kinase inhibitor erlotinib in two large phase III studies. Clin Cancer Res 2007;13:3913-21.

27. Bonner JA, Harari PM, Giralt J, et al. Radiotherapy plus cetuximab for locoregionally advanced head and neck cancer: 5-year survival data from a phase 3 randomised trial, and relation between cetuximab-induced rash and survival. Lancet Oncol 2010;11:21-8.

28. Cunningham D, Humblet $\mathrm{Y}$, Siena $\mathrm{S}$, et al. Cetuximab monotherapy and cetuximab plus irinotecan in irinotecan-refractory metastatic colorectal cancer. N Engl J Med 2004;351:337-45.

29. Bokemeyer C, Bondarenko I, Makhson A, et al. Fluorouracil, leucovorin, and oxaliplatin with and without cetuximab in the first-line treatment of metastatic colorectal cancer. J Clin Oncol 2009;27:663-71.

30. National institute for Health and Clinical Excellence. Appraising lifeextending, end of life treatments. http://www.nice.org.uk/media/E4A/ 79/SupplementaryAdviceTACEoL.pdf

31. Araújo A, Parente B, Sotto-Mayor R, et al. An economic analysis of erlotinib, docetaxel, pemetrexed and best supportive care as second or third line treatment of non-small cell lung cancer. Rev Port Pneumol 2008;14:803-27.

32. Carlson JJ, Reyes C, Oestreicher N, et al. Comparative clinical and economic outcomes of treatments for refractory non-small cell lung cancer (NSCLC). Lung Cancer 2008;61:405-15.

33. Thongprasert S, Tinmanee S, Permsuwan U. Cost-utility and budget impact analyses of gefitinib in second-line treatment for advanced non-small cell lung cancer from Thai payer perspective. Asia Pac J Clin Oncol 2012;8:53-61.

34. Wang S, Peng L, Li J, et al. A trial-based cost-effectiveness analysis of erlotinib alone versus platinum-based doublet chemotherapy as first-line therapy for Eastern Asian nonsquamous non-small-cell lung cancer. PLOS ONE 2013;8:e55917.

35. Chouaid C, Le Caer H, Corre R, et al. Cost analysis of erlotinib versus chemotherapy for first-line treatment of non-small-cell lung cancer in frail elderly patients participating in a prospective phase 2 study (GFPC 0505). Clin Lung Cancer 2013;14:103-7.

36. Cromwell I, van der Hoek K, Melosky B, et al. Erlotinib or docetaxel for second-line treatment of non-small cell lung cancer: a real-world cost-effectiveness analysis. J Thorac Oncol 2011;6:2097-103.

37. Fragoulakis VF, Pallis AG, Kaitelidou DK, et al. Economic evaluation of pemetrexed versus erlotinib as second-line treatment of patients with advanced/metastatic non-small cell lung cancer in Greece: a cost minimization analysis. Lung Cancer Targets Therapy Archives 2012;3:43-51.

38. Bradbury PA, Tu D, Seymour L, et al, on behalf of the NCIC Clinical Trials Group Working Group on Economic Analysis. Economic analysis: randomized placebo-controlled clinical trial of erlotinib in advanced non-small cell lung cancer. J Natl Cancer Inst 2010;102:298-306. 
39. PBAC. 07-2013: Afatinib, tablet, $20 \mathrm{mg}, 30 \mathrm{mg}, 40 \mathrm{mg}$ and $50 \mathrm{mg}$, (as dimaleate), Giotrifß (first line). http://www.pbs.gov.au/info/ industry/listing/elements/pbac-meetings/psd/2013-07/afatinib-first-line

40. NICE. TA192: Gefitinib for the first-line treatment of locally advanced or metastatic non-small-cell lung cancer. http://www.nice.org.uk/ guidance/TA192

41. NICE. TA124 (2007): Pemetrexed for the treatment of non-small-cell lung cancer. http://www.nice.org.uk/guidance/ta124

42. NICE. TA309 (2014): Pemetrexed maintenance treatment following induction therapy with pemetrexed and cisplatin for nonsquamous non-small-cell lung cancer. http://www.nice.org.uk/ guidance/TA309

43. NICE. TA296 (2013): Crizotinib for previously treated non-small-cell lung cancer associated with an anaplastic lymphoma kinase fusion gene. http://www.nice.org.uk/guidance/TA296
44. Pan Canadian Oncology Drug Review (pCODR). 2013. Crizotinib (Xalkori) Resubmission for Advanced Non-Small Cell Lung Cancer. http://www.pcodr.ca/wcpc/portal/Home/FindaReview/XalkoriAdv NSCLCResub?_afrLoop=457986569182000\&_afrWindowMode=0\& adf.ctrl-state=17jia3apey_276

45. Brown T, Pilkington G, Bagust $A$, et al. Clinical effectiveness and costeffectiveness of first-line chemotherapy for adult patients with locally advanced or metastatic non-small cell lung cancer: a systematic review and economic evaluation. Health Technol Assess 2015;17:281-2.

46. Chouaid C, Le Caer H, Locher C, et al. GFPC 0504 Team. Cost effectivenes of erlotinib versus chemotherapy for first-line treatment of non small cell lung cancer (NSCLC) in fit elderly patients participating in a prospective phase 2 study (GFPC 0504). BMC Cancer 2012;12:301.

47. http://www.nice.org.uk/newsroom/pressreleases/CrizotinibACD.jsp 


\section{Correction}

Khan I, Morris S, Hackshaw A, et al. Cost-effectiveness of first-line erlotinib in patients with advanced non-small-cell lung cancer unsuitable for chemotherapy. BMJ Open 2015;5:e006733. doi: 10.1136/bmjopen-2014-006733

In figure 1, the top square currently reads ' 370 Randomised'. This should read ' 670 Randomised' as mentioned earlier in the text.

Open Access This is an Open Access article distributed in accordance with the terms of the Creative Commons Attribution (CC BY 4.0) license, which permits others to distribute, remix, adapt and build upon this work, for commercial use, provided the original work is properly cited. See: http://creativecommons.org/licenses/by/4.0/

BMJ Open 2017;0:e006733corr1. doi:10.1136/bmjopen-2014-006733corr1 